\title{
Photooxidation of Chloride by Oxide Minerals: Implications for Perchlorate on Mars
}

\author{
Jennifer D. Schuttlefield ${ }^{\ddagger, \#}$, Justin B. Sambur ${ }^{ \pm, \pm}$, Melissa Gelwicks ${ }^{\ddagger}$, Carrick M. Eggleston \\ and B. A. Parkinson ${ }^{ \pm \pm \pm^{*}}$
}

\section{Full References:}

15. Smith, P. H.; Tamppari, L. K.; Arvidson, R. E.; Bass, D.; Blaney, D.; Boynton, W. V.; Carswell, A.; Catling, D. C.; Clark, B. C.; Duck, T.; DeJong, E.; Fisher, D.; Goetz, W.; Gunnlaugsson, H. P.; Hecht, M. H.; Hipkin, V.; Hoffman, J.; Hviid, S. F.; Keller, H. U.; Kounaves, S. P.; Lange, C. F.; Lemmon, M. T.; Madsen, M. B.; Markiewicz, W. J.; Marshall, J.; McKay, C. P.; Mellon, M. T.; Ming, D. W.; Morris, R. V.; Pike, W. T.; Renno, N.; Staufer, U.; Stoker, C.; Taylor, P.; Whiteway, J. A.; Zent, A. P. Science 2009, 325, 58-61.

18a. Squyres, S. W.; Grotzinger, J. P.; Arvidson, R. E.; Bell, J. F. III; Calvin, W.; Christensen, P. R.; Clark, B. C.; Crisp, J. A.; Farrand, W. H.; Herkenhoff, K. E.; Johnson, J. R.; Klingelhöfer, G.; Knoll, A. H.; McLennan, S. M.; McSween, H. Y. Jr.; Morris, R. V.; Rice, J. W. Jr.; Rieder, R.; and Soderblom, L. A. Science 2004, 306, 1709-1714. 